Cahiers québécois de démographie

Revue internationale d'étude des populations

\title{
La langue de travail au Québec en 2006 et 2016 : une réplique à Michel Paillé
}

\section{Jean-Pierre Corbeil}

Volume 48, numéro 2, automne 2019

URI : https://id.erudit.org/iderudit/1074183ar

DOI : https://doi.org/10.7202/1074183ar

Aller au sommaire du numéro

Éditeur(s)

Association des démographes du Québec

ISSN

1705-1495 (numérique)

Découvrir la revue

Citer ce document

Corbeil, J.-P. (2019). La langue de travail au Québec en 2006 et 2016 : une réplique à Michel Paillé. Cahiers québécois de démographie, 48(2), 229-231. https://doi.org/10.7202/1074183ar d'utilisation que vous pouvez consulter en ligne. 


\title{
Cahiers québécois de démographie
}

Vol. 48, n² 2, automne 2019, p. 229-231

\section{La langue de travail au Québec en 2006 et 2016 : une réplique à Michel Paillé}

\author{
JEAN-PIERRE CORBEIL*
}

e 29 novembre 2017, Statistique Canada diffusait les données officielles du recensement de
2016 portant sur la langue de travail. Dans le document Recensement en bref intitulé « Les langues de travail au Canada : Recensement de la population, 2016 », l'on y apprenait que, en 2016, 94,4 \% des travailleurs du Québec déclaraient faire une utilisation au moins régulière du français au travail, comparativement à 94,3\% en 2006.

Dans ce document analytique, le tableau 1 permettait aux lecteurs de constater que, de ce pourcentage, 56,5\% n'utilisaient que le français en 2016, 23,2\% y utilisaient surtout le français le plus souvent, mais y utilisaient aussi une autre langue de façon régulière (essentiellement l'anglais) en plus du français, que 7,4\% déclaraient utiliser le français à égalité avec une autre langue (essentiellement l'anglais) le plus souvent au travail et que, finalement, 7,2\% des travailleurs déclaraient utiliser le français de façon régulière comme langue secondaire au travail, c'est-à-dire en plus de celle qu'ils y utilisent le plus souvent. À la lumière des données de 2006, on y apprenait également que la proportion des travailleurs déclarant utiliser le français de façon prédominante au travail (uniquement + surtout) était donc passée de $\mathbf{8 2 , 0} \%$ à 79,7\%.

Il est étonnant de lire dans la note de M. Paillé qu'une telle présentation des résultats sur la langue de travail au Québec (lire une telle «typologie linguistique ») repose sur l'utilisation d'un " indicateur très rudimentaire » de l'utilisation du français «qui ne fait que compter sans tenir compte du contexte ». L'auteur ajoute en ce sens que les résultats "placent sur le même pied les réponses aux deux questions portant sur les langues de travail, ce qui a pour effet de minimiser leur différence » et de « leurrer » les utilisateurs de données sur la situation réelle du français en milieu de travail au Québec. Les données présentées lors de la diffusion du 29 novembre 2017 et reproduites ci-dessus nous semblent, au contraire, exprimer pleinement et avec rigueur les nuances et les distinctions qu'autorise la question de recensement en deux volets sur les langues utilisées au travail.

La prise en compte des personnes ayant déclaré faire une utilisation du français à égalité avec une autre langue (335 355 travailleurs au Québec en 2016) ou une utilisation régulière du français au travail en tant que langue secondaire (326 595 travailleurs en 2016) revient, selon l'auteur, à

* Ph. D., Directeur adjoint et responsable du Centre de la statistique ethnoculturelle, langue et immigration, Statistique Canada ; jean-pierre.corbeil@canada.ca 
accorder une importance à des " réponses marginales », voire à une utilisation " accessoire » du français. Selon le libellé de la question, et en particulier du volet B, tous ces travailleurs déclarent faire usage du français sur une base régulière au travail. Cette utilisation peut représenter jusqu'à $50 \%$ de leur utilisation d'une langue au travail.

Toutefois, le problème central identifié par l'auteur est que « faire la somme des occurrences consiste à compter autant de fois qu'il le faut toutes les personnes recensées qui ont révélé utiliser au travail le français, l'anglais ou des langues tierces, que ces langues apparaissent seules ou dans une énumération ». L'auteur fournit une définition du terme qui démontre avec éloquence que Statistique Canada ne compte pas les « occurrences ». En 2016, 4273935 personnes ont fait mention d'un usage au moins régulier du français au travail. Aucune personne n'a fait mention plus d'une fois du français. Ainsi, 4273935 personnes utilisent le français au travail. Il s'agit bien de "personnes », de travailleurs, et non d'occurrences.

Statistique Canada ne recommande nullement de procéder à l'addition des mentions d'utilisation du français, de l'anglais et des autres langues au travail tel que le propose la note. L'auteur affirme, au terme d'une telle addition, que l'approche de Statistique Canada manque de rigueur puisqu'elle se solde par des totaux atteignant $137,5 \%$ en 2006 et 139,8 \% en 2016, une situation qui constituerait une aberration « dans les nombreux ouvrages de statistique ». Suivant un tel raisonnement, il serait par exemple inexact d'affirmer que, en 2016 au Québec, 94,5\% des Québécois peuvent soutenir une conversation en français et que 49,1 \% des Québécois peuvent soutenir une conversation en anglais, sous prétexte que la somme totalise près de $144 \%$.

À quelle question tente-t-on de répondre ici? S'agit-il d'examiner comment a évolué l'utilisation du français au travail au Québec ou s'agirait-il plutôt d'établir combien il y a de travailleurs francophones dans la province, ces derniers étant définis par leur utilisation prédominante du français au travail? Que faire alors de ceux qui y utilisent plus d'une langue?

Le raisonnement proposé par M. Paillé découle principalement de l'adoption d'une perspective centrée sur des groupes linguistiques mutuellement exclusifs. Cette perspective permet difficilement de prendre en compte les dynamiques linguistiques complexes, les situations de bilinguisme, voire de plurilinguisme, et tend à présenter l'évolution des comportements et des pratiques linguistiques au travail comme un jeu à somme nulle. En d'autres termes, lorsque plus de personnes déclarent utiliser l'anglais au travail, le nombre de personnes qui déclarent utiliser le français diminue nécessairement d'autant. Or, la réalité est beaucoup plus complexe.

Les résultats présentés par Statistique Canada, loin d'être contradictoires, fournissent un portrait d'ensemble qui révèle l'ampleur de la coexistence des langues en milieu de travail, et ce, sans masquer la réalité. En d'autres termes, les lectrices et lecteurs du document de Statistique Canada ont tout-à-fait pu constater que l'utilisation prédominante du français au travail au Québec était passée de 82,0 \% en 2006 à 79,7 \% en 2016 pendant que celle de l'anglais passait de $12,5 \%$ à $12 \%$, et que l'utilisation du français à égalité avec une autre langue (l'anglais pour l'essentiel) passait de $4,7 \%$ à $7,4 \%$.

Il y a plusieurs façons de présenter les données sur la langue tirées des recensements canadiens. À des fins de simplification, Statistique Canada a souvent lui-même réparti, selon une tradition bien établie, les réponses multiples aux questions linguistiques entre groupes linguistiques, lorsque l'analyse s'y prêtait et que l'objectif était de mettre en lumière les différences entre groupes définis 
en fonction de caractéristiques spécifiques. Il continuera de le faire. Par ailleurs, l'analyse des données sur la langue de travail effectuée par Houle et Corbeil pour le compte de l'Office québécois de la langue française (2019), laquelle portait principalement sur le volet A de la question, est une autre façon d'analyser les données sur la langue de travail au Québec.

L'approche privilégiée par Statistique Canada lors de la diffusion officielle des données sur la langue de travail à l'automne 2017, soit celle portant sur l'utilisation du français, de l'anglais et des langues dites tierces, découle d'un intérêt grandissant parmi les utilisateurs de données envers des approches qui prennent mieux en compte les situations d'utilisations multiples des langues dans des contextes de contacts inter-linguistiques fréquents. Une telle présentation des résultats ne change en rien le fait que les utilisateurs ont toujours accès aux mêmes tableaux et données que ceux diffusés lors des recensements antérieurs. Le document d'à peine 8 pages diffusé par Statistique Canada ne représente donc qu'une minuscule partie de l'information rendue publique au même moment sur ce sujet.

\section{BIBLIOGRAPHIE}

HOULE, R. et J.-P. CORBEIL. 2019. Utilisation du français et de l'anglais au travail au Québec en 2016 : portrait d'ensemble des facteurs sociodémographiques, des secteurs d'emploi et des professions. Office québécois de la langue française, Montréal. 